\title{
GRAPTOLITOS DE LA FORMACIÓN SURI (ARENIG) EN EL SISTEMA DE FAMATINA, ARGENTINA
}

\author{
Blanca A. TORO' y Edsel D. BRUSSA ${ }^{2}$ \\ ' Consejo Nacional de Investigaciones Científicas y Técnicas (CONICET). Departamento \\ de Paleontología y Geología. IANIGLA. CRICYT. Parque General San Martín. 5500 \\ Mendozal, Argentina. \\ Consejo Nacional de Investigaciones Científicas y Técnicas (CONICET). Cátedra de \\ Estratigralía y Geología Histórica. Facultad de Ciencias Exactas. Físicas y Naturales. \\ Universidad Nacional de Córdoba. Avenida Vélez Sarsfield 299, 5000 Córdoba. Argentina.
}

Toro, B. A. y Brussa, E. D. 1997. Graptolitos de la Formación Suri (Arenig) en el Sistema de Famatina, Argentina. [Graptolites of the Suri Formation (Arenig), Famatina Range, Argentina]. Revista Española de Paleontologia, 12 (2), 175-184. ISSN 0213-6937.

\begin{abstract}
A biostratigraphic analysis is presented of the graptolite fauna from the Saladillo River gorge, with a stratigraphic section located to the southwest of the town of Angulos, La Rioja Province, Argentina. The vertical ranges are discussed of the various graptolite species identified. Two graptolite biozones are recognized: Baltograptus deflexus Biozone and Didymograptellus bifidus Biozone. These are now described for the first time from the Famatina Range, and constitute a record for the Arenig biostratigraphy of this range. The assemblages suggest an early- to mid- Arenig age for an informal member of silicified black shales with tuff partings as well as for the lower part of the overlying informal member of grey-bluish laminated shales. Correlations are proposed between these fossiliferous beds and equivalent outcrops in northwestern Argentina. Comparisons are made between the biozones recognized here and world-wide graptolite zones. Finally, some paleobiogeographical comments are presented.
\end{abstract}

Key words: Graptolites, biostratigraphy, Lower Ordovician, Arenig, Famatina Range, La Rioja Province, Argentina.

\section{RESUMEN}

En este trabajo se presenta el análisis bioestratigráfico de la graptofauna proveniente de la quebrada del río Saladillo, ubicada al suroeste de la localidad de Angulos, en la provincia de La Rioja, Argentina. Se discute el rango vertical de las especies de graptolitos identificadas y se reconocen dos biozonas: la Biozona de Baltograptus deflexus y la Biozona de Didymograptellus bificlus. Estas son descritas por primera vez en el Sistema de Famatina y constituyen un aporte al esquema de biozonación correspondiente al Arenig de esá región. Las mismas permiten asignar al miembro de pelitas negras silicificadas con intercalaciones de tobas y al tramo inferior del miembro de pelitas laminadas gris azuladas de la Formación Suri una edad equivalente al lapso Arenig inferior-medio. Asimismo, se propone la correlación de estos niveles fosilíferos con afloramientos equivalentes del noroeste argentino y la comparación de las biozonas reconocidas con aquellas establecidas en otras regiones del mundo. Finalmente, se realizan algunas observaciones acerca de las alïnidades paleobiogeográficás de la graptofauna estudiada.

Palabras clave: Graptolitos, bioestratigrafía, Ordovícico Inferior, Arenig, Sistema de Famatina, provincia de La Rioja, Argentina.

\section{INTRODUCCIÓN Y MARCO GEOLÓGICO}

El objetivo de este trabajo es dar a conocer una nueva fauna de graptolitos de la Formación Suri en la región del río Saladillo, flanco oriental de la sierra de Famatina, Departamento Famatina, provincia de La Rioja (Fig. 1). Este estudio se encuentra enmarcado en un proyecto de revisión paleontológica y estratigrálica de la Formación Suri, que abarcá, además del estudio de los graptolitos, un 


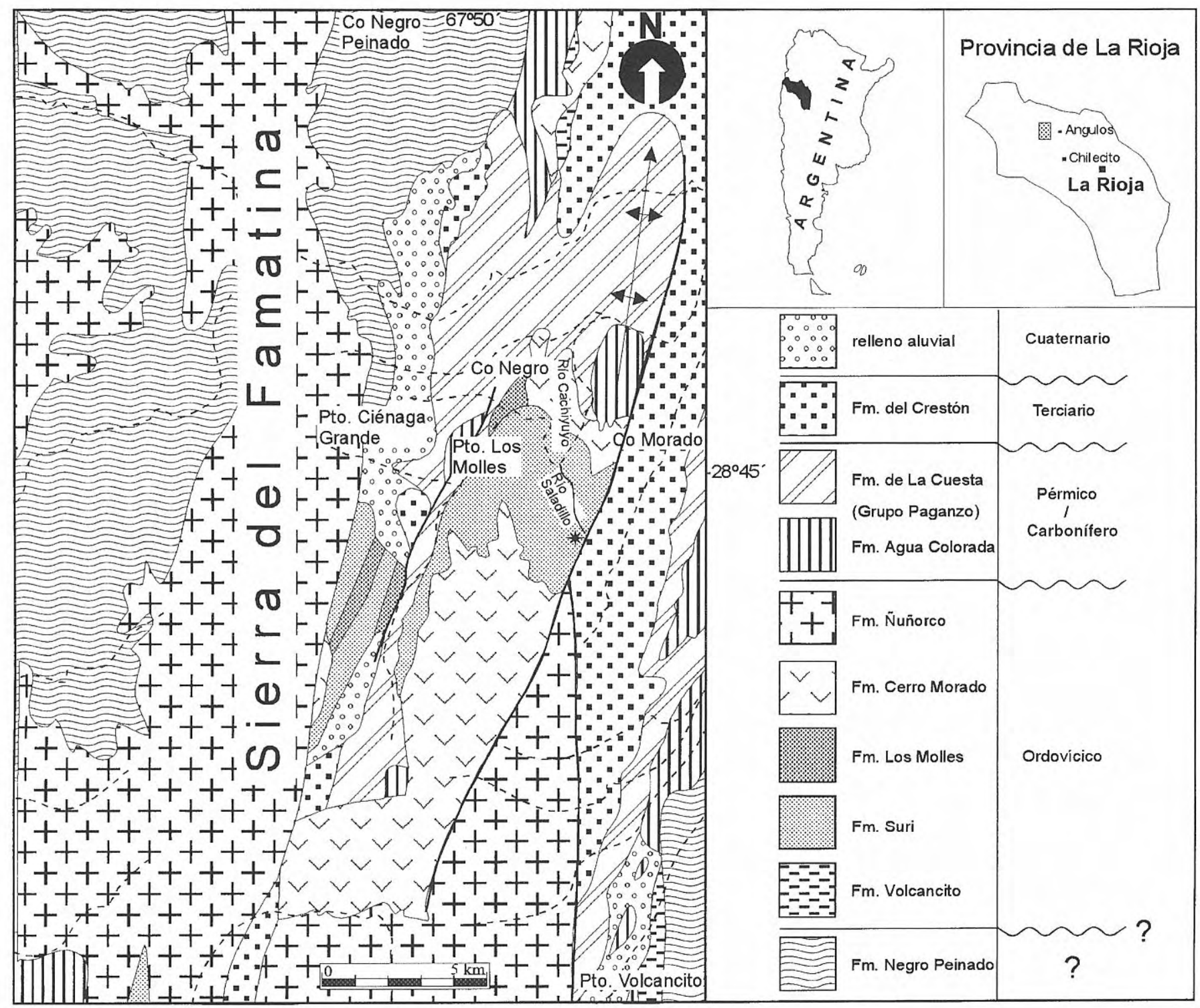

Figura 1. Mapa de ubicación de la quebrada del río Saladillo y distribución de los afloramientos de la Formación Suri (modificado de Turner, 1964 y Astini, datos inéditos). El asterisco indica la localización de los niveles fosilíferos.

estudio más completo de otros grupos fósiles como braquiópodos (Benedetto, en prep.), moluscos (Sánchez, en prensa) y trilobites (Vaccari y Waisfeld, en prep.).

El Sistema de Famatina se extiende entre las Sierras Pampeanas y la Precordillera en el noroeste de Argentina; este cinturón fue interpretado como un arco magmático complejo, típico de márgenes activos (Toselli et al., 1990, 1993; Sosa Gómez y Cisterna, 1994). Este sistema tiene más de $800 \mathrm{~km}$ de longitud si se considera que se extiende hacia el norte dentro de la Faja Eruptiva de la Puna y hacia el sur en las Sierras Pampeanas (sierra de Paganzo y probablemente sierra de Los Llanos). La hipótesis de la evolución del Sistema del Famatina como parte de un arco isla fue sugerida por diferentes autores (Aceñolaza y Toselli, 1984; Mannheim y Miller, 1992; Durand et al., 1994 entre otros). Posteriormente, y ya en un contexto regional, el arco volcánico del Famatina fue relacionado con la acreción del terreno de la Precordillera en el oeste de Gondwana, que habría tenido lugar en el Ordovícico Medio y Superior (Benedetto y Astini, 1993; Astini et al., 1995 y Astini y Benedetto, 1996).

La Formación Suri (Harrington en Harrington y Leanza, 1957) está compuesta, predominantemente, por areniscas silicificadas muy duras, lutitas de color verde, y limolitas con intercalaciones de bancos delgados de margas y tobas. Recientemente, Astini y Benedetto (1996) presentaron resultados preliminares sobre la sedimentología de esta unidad en el área estudiada. Estos autores reconocen una sección continua de más de 2000 metros que incluye las formaciones Suri y Los Molles. La primera unidad desarrolla 1400 metros; su base se encuentra limitada por una falla que la pone en contacto con la Formación del Crestón (Terciario); el techo pasa transicionalmente a la Formación Los Molles (Ordovícico). Los siguientes miembros informales fueron identificados de base a techo para la Formación Suri: a) 


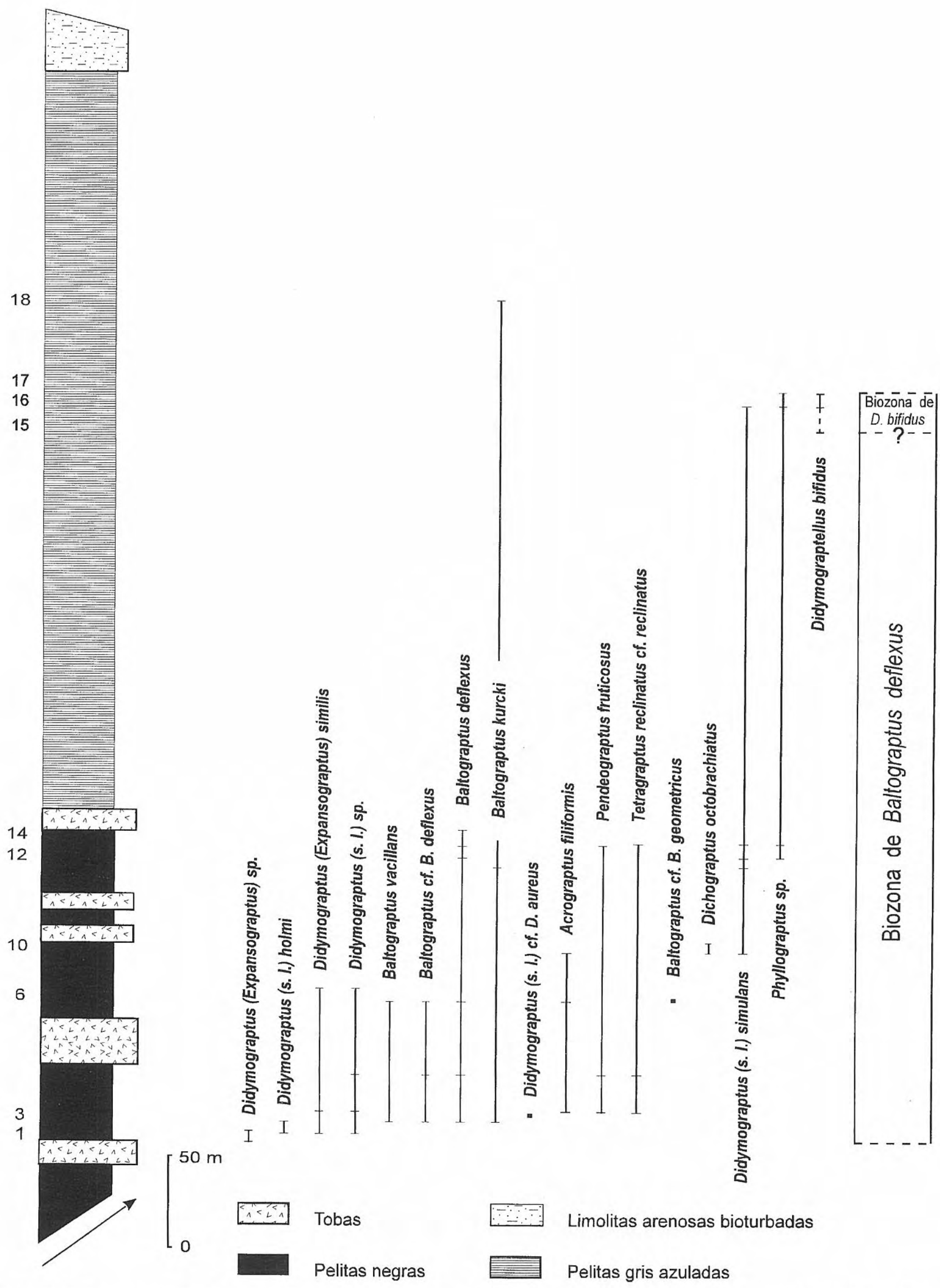

Figura 2. Columna estratigráfica de la Formación Suri con la distribución vertical de los taxones reconocidos y de las biozonas de graptolitos. Los números indican los principales horizontes fosilíferos. 
pelitas negras silicificadas con intercalaciones de tobas blanquecinas, b) pelitas laminadas gris azuladas, c) limolitas arenosas bioturbadas grises con nódulos calcáreos fosilíferos y d) areniscas y limolitas verdes bioturbadas con lentes de conchas.

Astini y Benedetto (1996) consideran que la sucesión puede ser interpretada como el relleno progresivo de una cuenca de retroarco con proveniencia tanto del arco como del antepaís. Este primer estadio estaría caracterizado por una rápida subsidencia, durante la que se depositan las pelitas negras con graptolitos en una cuenca restringida con escasas capas margosas hemipelágicas. Durante el Arenig inferior tuvo lugar una gran actividad volcánica que se manifiesta en los niveles inferiores de la Formación Suri, Biozona de Baltograptus deflexus, con la presencia de horizontes de tobas blanquecinas amarillentas laminadas y gradadas.

En la figura 2 se presenta la columna estratigráfica, la distribución vertical de las especies de graptolitos y las biozonas identificadas en los tramos inferiores de la Formación Suri (miembros de pelitas negras silicificadas con intercalaciones de tobas blanquecinas y de pelitas laminadas gris azuladas).

Los afloramientos portadores de la graptofauna analizada se encuentran $20 \mathrm{~km}$ al suroeste de la localidad de Angulos, a la que se accede por la Ruta Nacional $\mathrm{N}^{\circ}$ 40.

Las ilustraciones con cámara clara fueron realizadas en la Cátedra de Estratigrafía y Geología Histórica de la Facultad de Ciencias Exactas, Físicas y Naturales de la Universidad Nacional de Córdoba, donde se encuentra depositado el material paleontológico, bajo la sigla CEGH-UNC.

\section{ANTECEDENTES PALEONTOLÓGICOS}

Los trabajos previos en esta unidad, en el área de estudio, fueron referidos principalmente a trilobites. $\mathrm{La}$ única mención sobre la presencia de graptolitos en esta área corresponde a una comunicación personal de Harrington (en Turner, 1964). Inicialmente, los trilobites de la Formación Suri fueron estudiados por Harrington y Leanza (1957). Posteriormente, Aceñolaza y Rábano (1990) proporcionaron un listado de los taxones reconocidos en esta región y en el mismo son incluidos: Pliomera tmetophrys Harrington y Leanza, Merlinia megacantha (Leanza), Suriaspis cachiyuyana Aceñolaza y Rábano, Ogygitella australis Harrington y Leanza, Hungioides intermedius (Harrington y Leanza), Famatinolithus noticus Harrington y Leanza, Lonchodomas suriensis Harrington y Leanza y Ampyx cf. linleyensis Whittard. A partir de dicha asociación, estos autores sugirieron la posibilidad de una edad llanvirniana para la parte superior de la formación en el área del río Cachiyuyo, basada en la Zona de Merlinia megacantha, originalmente asignada a esta edad por Harrington (en Harrington y Leanza, 1957). En estudios previos, la edad de esta asociación había sido sugerida como arenigiana (Aceñolaza y Toselli, 1977, 1981).
El estudio llevado a cabo por Vaccari y Waisfeld (1994) sobre la fauna de trilobites en la secuencia volcaniclástica de la Formación Suri, aflorante en el tramo medio del río Chaschuil del flanco occidental de la Sierra de Narváez (Departamento Tinogasta, provincia de Catamarca), permitió distinguir dos asociaciones. Una inferior caracterizada por la presencia de Carolinites genacinaca Ross, Oopsites sp., Merlinia zupaya Vaccari y Waisfeld y Ampyx tinogastaensis Vaccari y Waisfeld, y una asociación superior compuesta por Annamitella longulosa Vaccari y Waisfeld, Neseuretus chaschuilensis Vaccari y Waisfeld y Merlinia megacantha (Leanza). La presencia de $C$. genacinaca permitió a estos autores postular una edad arenigiana temprana a media, la cual fue fijada con base en las graptofaunas asociadas a dicho taxón en el Miembro Olenidsletta de la Formación Valhallfonna de Spitsbergen (Fortey, 1975; Cooper y Fortey, 1982). El resto de los fósiles conchíferos estudiados son braquiópodos, bivalvos y rostroconchos (Benedetto, 1994; Sánchez, en prensa) cuyos taxones más significativos son mencionados en el apartado Consideraciones Paleobiogeográficas.

Recientemente, Albanesi y Vaccari (1994) describieron por primera vez conodontos de la Formación Suri en el tramo medio del río Chaschuil. La asociación está integrada por: Baltoniodus navis Lindström, Baltoniodus navis sensu Cooper, Chionoconus avangna Smith, Erraticodon patu Cooper, Jumudontus gananda Cooper, Protopanderodus rectus (Lindström), Trigonodus brevibasis (Sergeeva), Tropodus australis (Serpagli) y Semiacontiodus sp. Estos autores asignaron esta asociación a la Zona Baltoscandinava de Baltoniodus navis, correspondiéndole una edad arenigiana media.

\section{BIOESTRATIGRAFÍA}

Como resultado del estudio paleontológico de la graptofauna, se han identificado diecisiete taxones. La clasificación sistemática de los mismos está basada principalmente en los trabajos de Cooper y Fortey (1982), Williams y Stevens (1988) y Maletz (1994). Se ha mantenido el uso de morfogéneros (como Didymograptus sensu lato) para incluir especies cuya estructura proximal es desconocida, y del subgénero Expansograptus, hasta tanto se aclaren las relaciones filogenéticas de las especies incluídas en el mismo. En la figura 3 se presentan las ilustraciones de las formas más características.

El análisis de la distribución vertical de dichos taxones permitió establecer en forma preliminar, la existencia de dos nuevas biozonas para la Formación Suri: las Biozonas de Baltograptus deflexus y Didymograptellus bifidus (Fig. 2). La primera se desarrolla a través del miembro de pelitas negras silicificadas con intercalación de tobas blanquecinas y de la mitad inferior del miembro de pelitas laminadas gris azuladas, mientras que la Biozona de Didymograptellus bifidus se ubica en la porción superior de este último miembro. La presencia de ambas biozonas indicaría una 


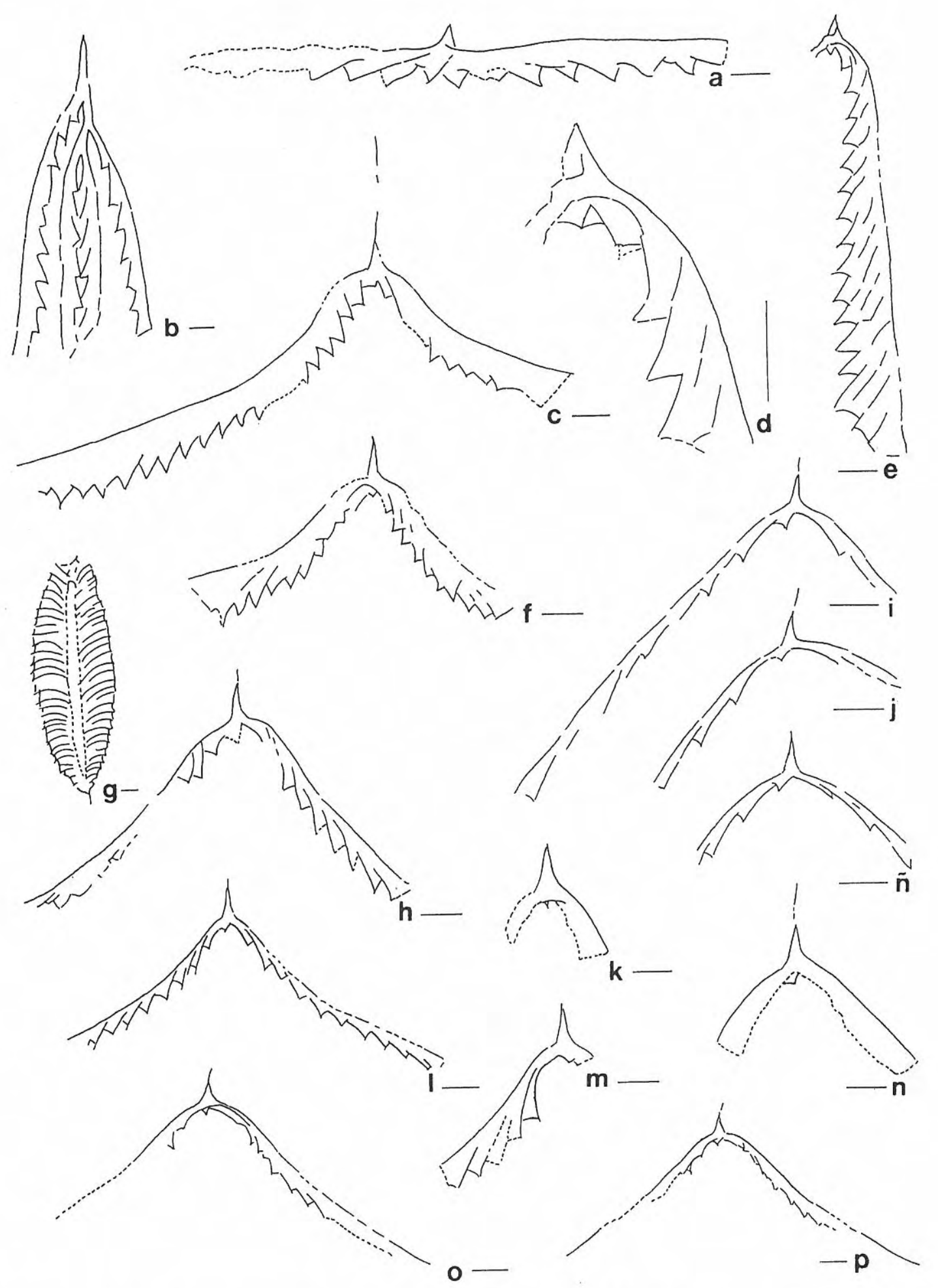

Figura 3. a, Didymograptus (Expansograptus) sp. Biozona de Baltograptus deflexus. CEGH-UNC 14.189. b, Pendeograptus fruticosus (J. Hall). Biozona de Baltograptus deflexus. CEGH-UNC 14.089. c, f, Baltograptus deflexus (Elles y Wood). Biozona de Baltograptus deflexus. c. CEGH-UNC 14.122. f. CEGH-UNC 14.117. d, e, Didymograptellus bifidus (J. Hall). Biozona de Didymograpellus bifidus. d. CEGH-UNC 14.211, detalle del extremo proximal. e. CEGH-UNC 14.211. g, Phyllograptus sp. Biozonas de Baltograptus deflexus y Didymograptellus bifidus. CEGHUNC 14.199. h, l, o, p, Baltograptus kurcki (Törnquist), Biozonas de Baltograptus deflexus y Didymograptellus bifidus. h. CEGH-UNC 14.282. 1. CEGH-UNC 14.097. o. CEGH-UNC 14.102. p. CEGH-UNC 14.104. i, j, ñ, Acrograptus filiformis (Tullberg), Biozona de Baltograptus deflexus. i. CEGH-UNC 14.181b. j. CEGH-UNC 14.181a. ñ. CEGH-UNC 14.180. k, m, n, Baltograptus vacillans (Tullberg), Biozona de Baltograptus deflexus. k. CEGH-UNC 14.069b. m. CEGH-UNC 14.070. n. CEGH-UNC 14.069a. (Escala gráfica = $1 \mathrm{~mm}$ ). 
edad arenigiana temprana a arenigiana media para estos niveles de la Formación Suri.

\section{Biozona de Baltograptus deflexus}

Esta biozona se extiende a través de, aproximadamente, 370 metros que corresponden al tramo inferior de la Formación Suri (Fig. 2). Su extensión total no puede precisarse debido a que la base de dicha unidad se encuentra truncada por fallamiento. El límite superior se ha hecho coincidir con la primera aparición de Didymograptellus bifidus (J. Hall) (Figura 2, horizonte fosilífero 15). La ubicación del mismo es tentativa debido a la escasez de graptolitos en la parte inferior del miembro de pelitas laminadas gris azuladas. Nuevos hallazgos de esta especie en niveles inferiores podrían modificar su ubicación definitiva. Esta biozona se encuentra caracterizada por el registro de la especie Baltograptus deflexus (Elles y Wood) y de numerosos taxones comúnmente encontrados en niveles correspondientes al Arenig Inferior. En su parte inferior se destaca la presencia de especies con rabdosomas de tipo extensiforme, como Didymograptus (Expansograptus) similis (J. Hall), Didymograptus (Expansograptus) sp., Didymograptus (s. 1.) holmi Törnquist, Didymograptus (s. 1.) cf. aureus T. S. Hall y Didymograptus (s. 1.) sp. Asimismo, es frecuente el registro de representantes del género Baltograptus, como B. vacillans (Tullberg), $B$. cf. deflexus y $B$. cf. geometricus (Törnquist), mientras que $B$. kurcki (Törnquist) continúa presente en la biozona suprayacente, al igual que Didymograptus (s. 1.) simulans Elles y Wood y Phyllograptus sp., los cuales comienzan a registrarse en la porción inferior a media de la biozona en cuestión. Las especies Acrograptus filiformis (Tullberg), Pendeograptus fruticosus (J. Hall), Tetragraptus reclinatus cf. reclinatus Elles y Wood y Dichograptus octobrachiatus (J. Hall) se encuentran restringidas también, en el Famatina, a la mitad inferior de esta biozona, y fueron localizadas anteriormente en niveles equivalentes de la Zona de Baltograptus deflexus, descrita en el borde occidental de la Cordillera Oriental argentina (Toro, 1994a y b, en prensa a y b).

La especie Baltograptus deflexus fue definida por Elles y Wood (1901) en la secuencia británica del Lake District, donde dicho taxón caracteriza la subzona homónima correspondiente al Arenig inferior. Posteriormente, Jackson (1962, 1978) y Molyneux y Rushton (1988) mencionan la presencia de Pendeograptus fruticosus, Acrograptus filiformis, B. aff. $B$. deflexus, B. aff. vacillans attenuatus Monsen y D. (E.) similis en dicha biozona, mientras que Cooper y Lindholm (1990) la equiparan al Bendigoniano (parte de Bendigoniano 2 a Bendigoniano 4) de Australia. Tanto la aparición del fósil guía como la asociación de taxones anteriormente descrita en esta biozona, permiten correlacionarla con la Subzona de $B$. deflexus de Inglaterra.

La existencia de formas expansográptidas, como Didymograptus (E.) similis y de otras como B. vacillans, A. filiformis, $P$. fruticosus, tetragráptidos reclinados y la aparición de representantes del género Phyllograptus, son características en la porción superior de la Zona de Didymograptus (s. 1.) balticus y parte inferior de la Zona de Pseudophyllograptus densus de Suecia (Cooper y Lindholm, 1990; Lindholm, 1991) y en la Zona de Didymograptus (s. 1.) balticus y parte inferior de la Zona de Pseudophyllograptus densus de Noruega (Monsen, 1937; Lindholm, 1991). Debido a ello, se sugiere una correlación entre la Biozona de $B$. deflexus, que aquí se describe, y las mencionadas biozonas, correspondientes al lapso Hunnebergiano tardío-Billingeniano temprano de la zonación baltoescandinava.

El hallazgo de $B$. deflexus en Argentina fue realizado por Loss (1951) en el extremo austral de la Cordillera Oriental. Este autor describe la especie en asociación con $B$. vacillans, en niveles equiparables al Arenig inferior del Cerro San Bernardo (provincia de Salta). Posteriormente, numerosos trabajos han hecho referencia a asociaciones de graptolitos del noroeste Argentino que incluyen dicho taxón. Una amplia discusión referida a la correlación local y regional de la Zona de $B$. deflexus descrita en el borde occidental de la Cordillera Oriental, fue brindada por Toro (1994a y b, en prensa a y b) y escapa a los objetivos de este trabajo. Cabe destacar que todas las especies que integran la asociación de la Zona de B. deflexus, reconocida en la Formación Suri, han sido encontradas anteriormente en los niveles correspondientes a dicha biozona en el borde occidental de la Cordillera Oriental, los cuales fueron asignados al Arenig inferior (parte del Be 2-Be 4 de la zonación australiana) por Toro (1994b, en prensa a y b). Con base en lo expuesto, se sugiere una edad equivalente para los niveles inferiores de la Formación Suri que contiene esta asociación. Asimismo, se propone su correlación con aquellos del tramo medio y parte del tramo superior de la Formación Acoite, aflorante en el borde occidental de la Cordillera Oriental, donde se identifica también la Zona de B. deflexus.

\section{Biozona de Didymograptellus bifidus}

La extensión asignada, en forma preliminar, a esta biozona es de aproximadamente 20 metros, correspondientes al tramo inferior de la Formación Suri, ubicado inmediatamente por encima de los niveles en los cuales se desarrolla la Biozona de Baltograptus deflexus.

Baltograptus kurcki y Didymograptus (s. 1.) simulans fueron localizados en las biozonas de B. deflexus y Didymograptellus bifidus en el borde occidental de la Cordillera Oriental (Toro, 1994b), mientras que representantes del género Phyllograptus fueron registrados sólo en la Zona de Didymograptellus bifidus (Toro, 1994a, en prensa a y b). La especie B. kurcki fue descrita en niveles correspondientes a la Zona de Pseudophyllograptus densus de Suecia y Noruega (Törnquist, 1901; Monsen, 1937), equiparada al Arenig inferior a medio por Cooper y Lindholm (1990). En Argentina fue localizada por Ramos (1974) en niveles asignados a la misma edad, en el borde oriental de la Puna y, posteriormente, Toro (1994b, en prensa a y b) cita su presencia en las Zonas de Tetragraptus 
akzharensis, Baltograptus deflexus y Didymograptellus bifidus, en el borde occidental de la Cordillera Oriental, correspondientes al lapso Arenig inferior a medio (Be 1Ch 2). Didymograptus (s. 1.) simulans fue definido por Elles y Wood (1901) en niveles de las Subzonas de Baltograptus deflexus y Didymograptus nitidus del Arenig inferior a medio de Gran Bretaña. Posteriormente, Zalasiewicz (1984, 1986) lo cita en el norte de Gales, y Williams (1990) amplía su registro al intervalo representado por las Zonas de Pendeograptus fruticosusIsograptus victoriae lunatus del oeste de Terranova y por las Zonas de B. deflexus-Isograptus gibberulus de Gran Bretaña. En Argentina, Rao et al. (1991) describen formas afines a esta especie y asignan los niveles portadores al Arenig inferior. Moya et al. (1994) mencionan ejemplares de Didymograptus (E.) simulans provenientes del tramo austral de la Cordillera Oriental, asignándolos al Chewtoniano, y recientemente, Toro (1994b, en prensa a y b) registra dicha especie en las Zonas de T. akzharensis, B. deflexus y D. bifidus (Arenig inferior a medio, equivalente a Be 1-Ch 2) del borde occidental de la Cordillera Oriental.

El límite inferior de la biozona que se discute se ha hecho coincidir, tentativamente, con la aparición de restos fosilíferos regularmente conservados, asignados a Didymograptellus cf. bifidus (Fig. 2, horizonte 15). Quince metros por encima, este registro es confirmado con ejemplares completos y bien preservados (horizonte 16). Didymograptellus bifidus fue descrito por J. Hall (1865) en el Arenig medio de Point Lévis, Québec (Canadá). Posteriormente, fue citado en niveles equivalentes en numerosas localidades, en su mayoría correspondientes a la Provincia Pacífica de graptolitos: lutita Deepkill, New York (Ruedemann, 1947); Marathon, Texas (Berry, 1960); Canning Basin, Australia (Legg, 1976) y en el suroeste de China (Mu et al., 1979). Cooper y Fortey (1982) lo registraron en la parte inferior de la Zona de Didymograptellus bifidus e Isograptus victoriae lunatus de Spitsbergen, en niveles correspondientes al Arenig medio. Más recientemente, Williams y Stevens (1988) lo describen en la Zona de Didymograptellus bifidus de Terranova.

La asociación graptolítica superior descrita en los niveles inferiores de la Formación Suri (Biozona de Didymograptellus bifidus), permite asignar a los mismos una edad arenigiana media (equivalente al lapso $\mathrm{Ch}$ 1-Ch 2 de Australia) y correlacionarlos con aquellos de la porción superior de la Formación Acoite, expuestos en las quebradas de Los Colorados y Chamarra en el borde occidental de la Cordillera Oriental (Toro, 1994a y b; en prensa a y b).

\section{CONSIDERACIONES PALEOBIOGEOGRÁFICAS}

La Formación Suri contiene asociaciones faunísticas de mezcla con representantes gondwánicos y 'precordilleranos' (Vaccari et al., 1993; Benedetto y Sánchez, 1996). Los trilobites de la Fauna de Neseuretus y braquiópodos del género Incorthis sugieren afinidades con la región occidental de Gondwana (Marruecos, Bolivia y noroeste de Argentina), mientras que la presencia de braquiópodos de los géneros Skenidioides y Monorthis indica afinidades célticas (Benedetto, 1994). Según Neuman y Harper (1992), las faunas con afinidades célticas se habrían desarrollado en la periferia de Gondwana, en latitudes medias a altas. Los bivalvos y rostroconchos indican sólo afinidades perigondwánicas y se encuentran asociados a formas endémicas (género Catamarcaia) y cosmopolitas (e.g. representantes de los géneros Redonia y Ribeiria) (Sánchez, en prensa).

Con respecto a la conodontofauna de la Formación Suri en el área de Chaschuil (Albanesi y Vaccari, 1994), los géneros Chionoconus, Erraticodon, Jumudontus y Tropodus son característicos de aguas cálidas y han sido localizados en las Provincias de Laurencia (e.g. Ethington y Repetski, 1984; Stait y Barnes, 1991), Siberia (e.g. Kanigin et al., 1989), China del Norte (An y Zheng, 1990) y Australia (Cooper, 1981), típicas del Dominio paleobiográfico "Midcontinent". Por otro lado, fueron identificados, además, géneros típicos de aguas frías, como Baltoniodus, Protopanderodus y Semiacontiodus. Estas formas han sido localizadas en las Provincias de Baltoscandia (e.g. Löfgren, 1978; Dzik, 1983) y China del Sur (e.g. An, 1987) correspondientes al Dominio Atlántico.

Cooper et al. (1991) sostienen que gran parte de los graptolitos del Ordovícico Inferior habitaron la zona epipelágica (entre 0 y 150-200 metros de profundidad) y no estuvieron sujetos a una zonación latitudinal. El análisis de la distribución de los taxones reconocidos en este estudio indica que, en su mayoría, corresponden a formas pandémicas que ocuparon el biotopo epipelágico. Entre ellas pueden mencionarse: Pendeograptus fruticosus, Dichograptus octobrachiatus, Didymograptus (E.) similis, D. (s. 1.) simulans, Tetragraptus reclinatus cf. reclinatus y Phyllograptus sp. Sin embargo, asociados a las formas pandémicas se han registrado Baltograptus deflexus, Didymograptellus bifidus y especies en común con Baltoscandia, como B. vacillans, B. kurcki, B. cf. geometricus y Acrograptus filiformis. Los taxones $B$. deflexus, $B$. vacillans y $B$. cf. deflexus indicarían que la graptofauna estudiada posee afinidades con la Provincia Atlántica de graptolitos, mientras que la aparición de Didymograptellus bifidus le confiere un carácter pacífico.

Este análisis resulta notablemente similar a lo observado en el borde occidental de la Cordillera Oriental (Toro, 1994b, 1995). Allí, la mezcla de afinidades, representada tanto en niveles correspondientes al Arenig inferior (Zonas de Tetragraptus phyllograptoides, T. akzharensis y $B$. deflexus) como al Arenig medio (Zona de $D$. bifidus) de la Formación Acoite, permitió postular a la autora que dicha región estuvo ubicada en la zona de transición de latitudes intermedias. El presente estudio sugiere una estrecha vinculación entre ambas regiones perigondwánicas durante el Arenig inferior-Arenig medio. Nuevos hallazgos fosilíferos en el área permitirían esclarecer las afinidades faunísticas con otras regiones del mundo, consideradas provincias de mezcla (como 
Baltoscandia y la provincia china de Yangtse), tal como fuera establecido en el borde occidental de la Cordillera Oriental (Toro, 1994b, 1995).

Finalmente, la ausencia de especies restringidas al biotopo de aguas profundas (en opinión de Cooper et al., 1991) indicaría que la graptofauna estudiada habría ocupado probablemente el biotopo epipelágico.

\section{AGRADECIMIENTOS}

Los autores desean dejar constancia de su agradecimiento a los árbitros, Dres. Juan Carlos GutiérrezMarco y Philippe Legrand, ya que con sus acertadas sugerencias permitieron mejorar el manuscrito. Para la realización de este trabajo se ha contado con el apoyo económico brindado por el Consejo Nacional de Investigaciones Científicas y Técnicas (CONICET), por el Consejo de Investigaciones Científicas y Tecnológicas de la Provincia de Córdoba (CONICOR) y por la Secretaría de Ciencia y Tecnología (SeCyT). Agradecemos a los colegas de la Cátedra de Estratigrafía y Geología Histórica de la Universidad Nacional de Córdoba la colección del material fosílifero.

\section{BIBLIOGRAFÍA}

Aceñolaza, F. G. y Rábano, I. 1990. Nota sobre algunos trilobites Asaphina de la Formación Suri (Sierra de Famatina, La Rioja, Argentina). Actas V Congreso Argentino de Paleontología y Bioestratigrafía, Tucumán, 1, 39-49.

Aceñolaza, F. G. y Toselli, A. J. 1977. Observaciones geológicas y paleontológicas sobre el Ordovícico de la zona de Chaschuil, provincia de Catamarca. Acta Geológica Lilloana, 14, 55-81.

Aceñolaza, F. G. y Toselli, A. J. 1981. Geología del Noroeste Argentino. Publicación especial de la Facultad de Ciencias Naturales, Universidad Nacional de Tucumán, 1287, 212 pp.

Aceñolaza, F. G. and Toselli, A. J. 1984. Lower Ordovician volcanism in North West Argentina. In: Aspects of the Ordovician System (Ed. D. L. Bruton), Palaeontological Contributions of the University of Oslo, 295, 203-209.

Albanesi, G. L. y Vaccari, N. E. 1994. Conodontos del Arenig en la Formación Suri, Sistema del Famatina, Argentina. Revista Española de Micropaleontología, 26 (2), 125-146.

An, T. 1987. The Lower Paleozoic conodonts of South China. Peking University Publication House, Beijing, $238 \mathrm{pp}$.

An, T. and Zheng, Z. 1990. The conodonts of the marginal areas around the Ordos basin, North China. Science Press, $201 \mathrm{pp}$.

Astini, R. A. and Benedetto, J. L. 1996. Paleoenvironmental features and basin evolution of a complex volcanic arc region in the pre-andean western Gondwana: the Famatina belt. III International Symposium on Andean Geodynamics, St. Melo, Francia, 755-758.
Astini, R. A., Benedetto, J. L. and Vaccari, N. E. 1995. The Early Paleozoic evolution of the Argentine Precordillera as a Laurentian rifted, drifted and collided terrane: A Geodynamic model. Geological Society of America Bulletin, 107, 253-273.

Benedetto, J. L. 1994. Braquiópodos ordovícicos (Arenigiano) de la Formación Suri en la región del río Chaschuil, Sistema de Famatina, Argentina. Ameghiniana, 31, 221-238.

Benedetto, J. L. and Astini, R. A. 1993. A collisional model for the stratigraphic evolution of the Argentine Precordillera during the Early Paleozoic. II International Symposium on Andean Geodynamic, Oxford, 501-504.

Benedetto, J. L and Sánchez, T. M. 1996. Paleobiogeography of brachiopods and molluscan faunas along the South American margin of Gondwana during the Ordovician. In: El Paleozoico Inferior en el Noroeste de Gondwana (Eds. B. Baldis y F. Aceñolaza). Serie de Correlación Geológica, 12, 23-38.

Berry, W. B. N. 1960. Graptolites faunas of the Marathon Region, West Texas. University of Texas Publication, 6005, 179 pp.

Cooper, B. J. 1981. Early Ordovician conodonts from the Horn Valley Siltstone, central Australia. Palaeontology, 24, 147-183.

Cooper, R. A. and Fortey, R. A. 1982. The Ordovician graptolites of Spitsbergen. Bulletin of the British Museum (Natural History) Geology, 36, 157-302.

Cooper, R. A. and Lindholm, K. 1990. A precise worldwide correlation of early Ordovician graptolite sequences. Geological Magazine, 127, 497-525.

Cooper, R. A., Fortey, R. A. and Lindholm, K. 1991. Latitudinal and depth zonation of early Ordovician graptolites. Lethaia, 24, 199-218.

Durand, F. R., Toselli, A. J., Aceñolaza, F. G. y Rossi de Toselli, J. N. 1994. Evolución Geológica del Sistema del Famatina durante el Precámbrico y Paleozoico. Instituto Superior de Correlación Geológica, Tucumán, Serie Monográfica y Didáctica, 14, 75 pp.

Dzik, J. 1983. Relationships between Ordovician Baltic and North American Midcontinent conodont faunas. Fossils and Strata, 15, 59-85.

Elles, G. L. and Wood, E. M. R. 1901. A monograph of British graptolites. Palaeontographical Society of London, Monograph, 171, 539 pp.

Ethington, R. and Repetski, J. 1984. Paleogeographic distribution of Early Ordovician conodonts in central and western United States. In: Conodont biofacies and provincialism. Special Paper of the Geological Society of America, 196, 89-101.

Fortey, R. A. 1975. The Ordovician trilobites of Spitsbergen. II. Ashapidae, Nileidae, Raphiophoridae and Telephinidae of the Valhallfonna Formation. Norsk Polarinstitutt Skrifter, 162, $125 \mathrm{pp}$.

Hall, J. 1865. Graptolites of the Quebec Group. Geological Survey of Canada, 2, $151 \mathrm{pp}$.

Harrington, H. G. and Leanza, A. F. 1957. Ordovician trilobites of Argentina. Department of Geology, University of Kansas, Special Publication, 1, 1-276. 
Jackson, D. 1962. Graptolites Zones in the Skiddaw Group in Cumberland, England. Journal of Paleontology, 36, 300-313.

Jackson, D. 1978. The Skiddaw Group. In: The Geology of the Lake District (Ed. F. Moseley). Yorkshire Geological Society, Occasional Publication, 3, 79-91.

Kanigin, A. V., Moskalenko, T. A., Yodrenkina, A. G., Abaimova, G., Semenova, V. C., Sichev, O. V. y Timogin, A. V. 1989. El Ordovícico de la Plataforma Siberiana (en ruso). Nauka Novosibirsk, 1-214.

Legg, D. P. 1976. Ordovician trilobites and graptolites from the Canning Basin, Western Australia. Geologica et Palaeontologica, 10, 1-58.

Lindholm, K. 1991. Hunnebergian graptolites and biostratigraphy in southern Scandinavia. Lund Publications in Geology, 95, 1-35.

Löfgren, A. 1978. Arenigian and Llanvirnian conodonts from Jämtland, Northern Sweden. Fossils and Strata, 13, 1-129.

Loss, R. 1951. Contribuciones al conocimiento de las faunas graptolíticas del Norte argentino. 1: Graptolites del Cerro San Bernardo (Salta) y zona del dique de La Ciénaga (Jujuy). Revista de la Asociación Geológica Argentina, 6, 21-61.

Maletz, J. 1994. Pendent didymograptids (Graptoloidea, Dichograptacea). In: Graptolite Research Today (Eds. X. Chen, B.-D. Erdtmann and Y. Ni). Nanjing University Press, 27-43.

Mannheim, R. y Miller, H. 1992. Sistema de Famatina, Noroeste de Argentina: acreción de un arco volcánico en el margen occidental del Gondwana en el Paleozoico Inferior. Publicaciones del Museo de Geología de Extremadura, 2, 102-103.

Monsen, A. 1937. Die graptolithenfauna in Unteren Didymograptusschiefer (Phyllograptusschiefer) Norwegens. Norsk Geologisk Tidsskrift, 16, 57-267.

Molyneux, S. G. and Rushton, A. W. A. 1988. The age of the Watch Hill Grits (Ordovician), English Lake District: structural and palaeogeographical implications. Transactions of the Royal Society of Edinburgh: Earth Sciences, 79, 43-69.

Moya, M. C., Malanca, S., Monteros, J. A. y Cuerda, A. 1994. Bioestratigrafía del Ordovícico en la Cordillera Oriental Argentina basada en graptolitos. Revista Española de Paleontología, 9, 91-104.

Mu, E., Ge, M., Chen, X., Ni, Y. and Lin, Y. 1979. Lower Ordovician graptolites of Sowthwest China. Palaeontologica Sinica, 156, 1-192.

Neuman, R. B. and Harper, D. A., 1992. Paleogeographic significance of Arenig - Llanvirn Toquima-Table Head and Celtic brachiopod assemblages. In: Global perspectives on Ordovician Geology (Eds. P. Webby and J. R. Laurie). Balkema, 241-254.

Ramos, V. A. 1974. Los didymográptidos de la Formación Parcha en el borde oriental de la Puna salteña. Revista de la Asociación Geológica Argentina, 29 (1), 73-84.

Rao, R. I., Hünicken, M. A. y Ortega, G. 1991. Conodontes y graptolitos ordovícicos en la quebrada de Los Colorados (Departamento de Tumbaya), Cordillera
Oriental, Provincia de Jujuy, Argentina. Anales de la Academia Brasilera de Ciencias, 63 (2), 185-192.

Ruedemann, R. 1947. Graptolites of North America. Memoir of the Geological Society of America, 19, $652 \mathrm{pp}$.

Sánchez, T. M. (en prensa). Bivalvia and Rostroconchia (Mollusca) from the Suri Formation, Early Ordovician (Arenig), Western Argentina. Journal of Paleontology.

Sosa Gómez, J. y Cisterna, C. E. 1994. Sistema de Famatina: arco magmático heterogéneo durante el Paleozoico Inferior. Serie Correlación Geológica. Resúmenes, Tucumán, 78-79.

Stait, K. A. and Barnes, C. R. 1991. Conodont biostratigraphy of the upper St. George Group (Canadian of Whiterockian), western Newfoundland. In: Advances in Ordovician Geology (Eds. C. R. Barnes and S. H. Williams). Geological Survey of Canada, 90(9), 125134.

Törnquist, S. L. 1901. Researches into the graptolites of the Lower Zones of Scanian and Vestrogothian PhylloTetragraptus Beds. Part 1. Lunds Universitets Arsskrift, 37, 2 (5), 1-26.

Toro, B. A. 1994a. Las zonas de Didymograptus (Didymograptellus) bifidus (Arenigiano medio) y Didymograptus (Corymbograptus) deflexus (Arenigiano inferior) en la Formación Acoite, Cordillera Oriental, Argentina. Ameghiniana, 31, 209-220.

Toro, B. A. 1994b. Taxonomía, bioestratigrafía y afinidades paleobiogeográficas en base a las graptofaunas ordovícicas del borde occidental de la Cordillera Oriental, provincia de Jujuy, Argentina. Tesis Doctoral en Ciencias Geológicas, Facultad de Ciencias Exactas, Físicas y Naturales, Universidad Nacional de Córdoba, 173 pp. (inédito).

Toro, B. A. 1995. Biostratigraphical paleoenvironmental aspects and paleogeographic affinities of the Arenigian graptolite fauna from the western flank of Cordillera Oriental, Argentina. Graptolite News, 8, 61-62.

Toro, B. A. (en prensa a). La fauna de graptolitos de la Formación Acoite, en el borde occidental de la Cordillera Oriental Argentina. Parte 1: Análisis bioestratigráfico. Ameghiniana.

Toro, B. A. (en prensa b). Análisis bioestratigráfico y paleoambiental de la graptofauna arenigiana de la Cordillera Oriental de Jujuy, Argentina. Revista del Instituto Argentino de Nivología y Glaciología.

Toselli, A., Saavedra, J., Pellitero, E., Rossi de Toselli, J., Aceñolaza, F y Medina, M. 1990. Geoquímica y petrogénesis del volcanismo ordovícico de la Formación Las Planchadas, Sistema de Famatina. Revista de la Asociación Geológica Argentina, 45, 313-322.

Toselli, A., Toselli de Rosi, J., Pellitero, E y Saavedra, J. 1993. El arco magmático granítico del Paleozoico Inferior del Sistema del Famatina, Argentina. XII Congreso Geológico Argentino, 4, 7-15.

Turner, J. C. 1964. Descripción geológica de la Hoja 15c. Vinchina, Provincia de La Rioja. Boletín del Servicio Geológico de la República Argentina, 100, 81 pp.

Vaccari, N. E. y Waisfeld, B. G. 1994. Nuevos trilobites de la Formación Suri (Ordovícico temprano) en la región de 
Chaschuil, Provincia de Catamarca. Implicancias bioestratigráficas. Ameghiniana, 31 (1), 73-86.

Vaccari, N. E., Benedetto, J. L., Waisfeld, B. G. y Sánchez, T. M. 1993. La fauna de Neseuretus en la Formación Suri (Oeste de Argentina): edad y relaciones paleobiogeográficas. Revista Española de Paleontología, 8, 185190.

Williams, S. H. 1990. An Arenig graptolite from Bell Island, eastern Newfoundland - its biostratigraphic and paleogeographic significance. Atlantic Geology, 26, 4355 .
Williams, S. H. and Stevens, R. K. 1988. Early Ordovician (Arenig) graptolites of the Cow Head Group, western Newfounland, Canada. Palaeontographica canadiana, 5, 1-167.

Zalasiewicz, J. A. 1984. A re-examination of the type Arenig Series. Geological Journal, 19, 105-124.

Zalasiewicz, J. A. 1986. Graptolites from the type Arenig Series. Geological Magazine, 123 (5), 537-544.

Manuscrito recibido: 12 de julio, 1996 Manuscrito aceptado: 20 de enero, 1997 